\title{
Autonomic Nervous System Functioning Associated with Epileptic Seizures: Analysis of Heart Rate Variability
} \author{
and Cluitmans PJM $^{1,4}$ \\ ${ }^{1}$ Epilepsy Centre Kempenhaeghe, Heeze, Netherlands \\ ${ }^{2}$ School for Mental Health and Neuroscience, Maastricht University, Maastricht, Netherlands \\ ${ }^{3}$ Reference Centre for Refractory Epilepsy, Department of Neurology, Ghent University Hospital, Ghent, Belgium \\ ${ }^{4}$ University of Technology, Eindhoven, the Netherlands Department of Neurology, Netherlands \\ ${ }^{5}$ Radiology, Maastricht University Medical Centre, Maastricht, the Netherlands \\ ${ }^{6}$ Maastricht University Medical Centre, Maastricht, the Netherlands
}

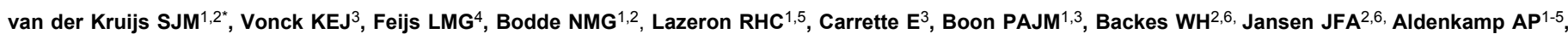

*Corresponding author: van der KruijsKempenhaeghe SJM, Epilepsy Centre Kempenhaeghe, Heeze, Netherlands, Tel: +31 (0)402279502; Fax: +31 (0)402265691; Email: sylvie.kolfschoten@gmail.com

Received date: May 27, 2014; Accepted date: June 24, 2014; Published date: June 30, 2014

Copyright: (c) 2014 van der Kruijs SJM, et al. This is an open-access article distributed under the terms of the Creative Commons Attribution License, which permits unrestricted use, distribution, and reproduction in any medium, provided the original author and source are credited.

\begin{abstract}
Objective: Little is known about the (peri-) ictal changes in autonomic nervous system activity of epileptic seizures. Such information may be useful for seizure prediction paradigms and differential diagnosis between epilepsy and psychogenic non-epileptic seizures (PNES). The current study investigated the peri-ictal time course of heart rate variability (HRV) measures, which reflect autonomic nervous system functioning.

Methods: HRV measures were extracted from electrocardiography data collected during 1-7 days of videoelectroencephalography monitoring of 17 patients with epilepsy and 20 patients with PNES. Heart rate (HR) and HRV measures (standard deviation of average beat-to-beat intervals (SDANN), root mean square of successive differences (RMSSD), high frequency (HF) power, low frequency (LF) power and very low frequency (VLF) power) were averaged over consecutive five-minute intervals. Quantitative analyses of Poincaré plot parameters (SD1, SD2 and SD1/SD2 ratio) were also performed. In addition, differences with HRV parameters of patients with PNES were explored.
\end{abstract}

Results: In epilepsy, no significant pre-ictal changes in HR and HRV parameters were observed. During seizures, HR, SDANN, SD1 and SD1/SD2 ratio significantly increased while VLF power significantly decreased. In the five-minute interval immediately following seizures, HR, SDANN and SD1 were back to pre-seizure levels, while VLF power remained significantly decreased and SD1/SD2 ratio remained significantly increased. Significant between-group differences were identified for several pre-ictal and ictal HRV parameters, but not for post-ictal measurements.

Conclusion: The ictal HR and HRV changes reflect increased sympathetic system activation during epileptic seizures. The HRV parameters of patients with epilepsy differed significantly from the peri-ictal HRV pattern of patients with PNES, which suggested increased sympathetic system activation and decreased vagal tone shortly before PNES and return to normal levels shortly after the episode. Implications for differential diagnosis and treatment are discussed.

Keywords: Epilepsy; Psychogenic; Non-epileptic seizures; Heart rate variability; Vagal tone; Autonomic nervous system

\section{Introduction}

Epileptic seizures result from brief episodes of abnormal excessive and synchronous neuronal activity in the brain. Examination of heart rate variability (HRV) parameters, which reflect (co-)activation of the sympathetic and parasympathetic branches of the autonomic nervous system in epilepsy, has previously identified altered sympathetic and vagal tone during interictal and ictal states [1-7]. These findings may have important meaning for our insight in the prognosis of epilepsy. For example, the ictal reduction of the vagal tone that has been repeatedly observed has been suggested to be one of the reasons for sudden unexpected death in epilepsy (SUDEP) [8,9].

A more detailed analysis of how HRV parameters change in the immediate pre-ictal period may be useful for seizure prediction paradigms, which could be used in vagal nerve or deep brain stimulators or for the purpose of real time seizure detection in physiological monitoring devices [6]. Therefore, the current study examined the peri-ictal HRV pattern associated with epileptic seizures. In addition, we investigated peri-ictal HRV patterns of psychogenic non-epileptic seizures (PNES), which are epilepsy-like episodes that lack epileptiform brain activity on the electroencephalogram (EEG), but instead originate from psychogenic factors [10,11]. Differences between the peri-ictal patterns of HRV changes in epilepsy and PNES 
were explored, in order to examine whether HRV measures could be useful for differential diagnosis.

\section{Materials and Methods}

\section{Study population}

The study population consisted of 17 patients with epilepsy (4M/ $13 \mathrm{~F})$, who were compared with a group of 20 patients with PNES (2M/ $18 \mathrm{~F})$. All patients had been previously monitored with video-EEG for 1-7 consecutive days in the Reference Centre for Refractory Epilepsy of Ghent University Hospital in Belgium. The diagnoses of epilepsy and PNES were based on the analysis of at least two episodes captured on video-EEG by two epileptologists with extensive experience in this field ( $>10$ years experience). Patients with dual pathology (epilepsy and PNES), were also included in the PNES group when epileptic seizures were controlled and when the PNES were confirmed by videoEEG monitoring. Exclusion criteria were uncertainty about the diagnosis and comorbid psychiatric disorders (e.g. mood and anxiety disorders, schizophrenia and psychosis, and substance-related disorders). The investigation received ethical approval by the Medical Ethical Committee of Ghent University.

\section{Electrocardiogram recordings}

The ECG was recorded throughout the video-EEG monitoring period (1-7 days) with a two-lead channel of the Micromed EEG system (Micromed S.p.A., Mogliano Veneto, Treviso, Italy). For the monitoring period, patients were in supine position in bed or on a chair during the day, and lying down in bed during the night. The start and end of epileptic seizures were defined by an epileptologist based on epileptiform brain activity on the EEG. The start and end of a PNES episode were defined by the associated motor symptoms (verified by the video recordings), which were often accompanied by unresponsiveness.

\section{Heart rate variability analysis}

HRV measures were derived according to the recommendations of the task force of the European Society of Cardiology and the North American Society of Pacing and Electrophysiology [12]. ECG data were exported in European Data Format (EDF, [13]) from the Micromed reporting system. These EDF files were imported in Matlab (Mathworks Inc., Natick, MA, USA). Custom-built Matlab scripts were used to subsequently carry out R-peak detection, model-based validation and - if necessary - correction of the list of subsequent RR intervals (tachogram) and HRV parameter calculation.

Two time-domain measures of HRV were calculated: SDANN (standard deviation of the average beat-to-beat intervals) and RMSSD (square root of the mean squared difference of successive beat-to-beat intervals). SDANN in general reflects the overall cyclic nature of HRV (the more sinusoidal the tachogram is, the higher SDANN), and is considered to be a measure for overall variability. RMSSD is considered to be a measure for vagal control of heart rate but also includes respiratory sinus arrhythmia, i.e., the local, intra-thoracic effect in HR fluctuations caused by respiratory pressure change during breathing.

Frequency-domain measures of HRV were calculated, using Fast Fourier Transformation to derive the spectral distribution. Indices included high-frequency (HF) power $(0.15-0.40 \mathrm{~Hz})$, low-frequency
(LF) power (.04-.15 Hz), and very low frequency (VLF) power (3-30 $\mathrm{mHz}$ ). Efferent vagal activity is a major contributor to the HF component, as has been consistently demonstrated by clinical and experimental observations [14-16]. Consequently, HF power can be seen as a reliable index of parasympathetic nervous system activity. The interpretation of the LF component is more controversial; some studies suggest LF power to be a marker of sympathethic modulation [16-19], while others conclude LF power to be a parameter that includes both sympathetic as vagal influences $[14,20]$. As a result, it is unclear whether the ratio of the latter two indices (LF/HF), can be regarded as a measure of sympathovagal balance or of sympathetic modulations. The physiological correlates of lower frequency components (VLF) of HRV are still unknown [12].

Quantitative analyses of Poincaré plot parameters were also performed. The Poincaré plot is a plot of $R R(n)$ on the $x$-axis versus $R R(n+1)$ on the $y$-axis; it plots the duration of each $R R$ interval against the duration of the next RR interval. The technique most commonly used to quantify a Poincaré plot is fitting an ellipse to the plot [21-24]. We obtained the standard deviation of instantaneous inter-beat interval (IBI) variability, SD1 (which measures the width of the Poincaré cloud) [24-27], the standard deviation of continuous longterm IBI variability, SD2 (which measures the length of the Poincaré cloud) [24-27] as well as the SD1/SD2 ratio. SD1, as a measure of instantaneous changes in IBI, is assumed to reflect vagal efferent activity [24], whereas SD2 expresses the overall variability in heart rate [28].

\section{Statistical analysis}

To examine patterns of HRV measures circa 1 hour before and circa 1 hour after seizures, twelve intervals (of approximately 5 minutes each) that preceded and followed the seizures were selected. Since it was not possible to select $25-26$ intervals in total for all seizures (12 pre-ictal intervals, 1-2 ictal intervals, and 12 post-ictal intervals) due to the occurrence of another seizure in a short time window or the removal of intervals which were disturbed by artefacts, $n$ differed per interval. Therefore, conservative non-parametric tests were used. To prevent patients with a high number of seizures from dominating the average, the intervals were first averaged per patient before inclusion in the within-subject analysis, resulting in an $n=17$ for the epilepsy group and $n=20$ for the PNES group. After visual inspection of the plotted averages of the HRV parameters, related-samples Wilcoxon signed-rank tests were used to test for statistical differences between all selected intervals. To assess differences between patients with epilepsy and patients with PNES for HR and HRV parameters, independentsamples Mann-Whitney $U$ tests were carried out. P-values $<0.05$ were considered statistically significant.

\section{Results}

\section{Demographic and clinical characteristics}

The study population included 17 patients with epilepsy (13 women, 4 men, age $37.7 \pm 17.4$, number of seizures during registration $4.7 \pm 3.0)$, with a seizure total of 80 , who were compared with 20 patients with PNES (18 women, 2 men, age $35.7 \pm 10.4$, number of seizures during registration $5.9 \pm 5.7$ ), who had a total number of 118 PNES episodes during video-EEG monitoring. Demographic information and clinical characteristics of the patients with epilepsy are summarized in Table 1. 
Citation: van der Kruijs SJM, Vonck KEJ, Feijs LMG, Bodde NMG, Lazeron RHC, et al. (2014) Autonomic Nervous System Functioning Associated with Epileptic Seizures: Analysis of Heart Rate Variability. J Neurol Neurophysiol 5: 215. doi:10.4172/2155-9562-5-1000215

Page 3 of 7

\begin{tabular}{|c|c|c|c|c|}
\hline ID & Age & Gender & $\begin{array}{l}\text { Nr. seizures } \\
\text { during registration }\end{array}$ & Type of epilepsy \\
\hline 1 & 11 & $\mathrm{~F}$ & 2 & Simple partial seizures (right frontal lobe origin) \\
\hline 2 & 18 & $\mathrm{~F}$ & 3 & Complex partial seizures (left frontal or temporal origin) \\
\hline 3 & 16 & $\mathrm{~F}$ & 8 & Simple partial seizures (left frontal lobe origin) \\
\hline 4 & 66 & $\mathrm{~F}$ & 5 & Primary generalized epilepsy (absence seizures) \\
\hline 5 & 58 & $\mathrm{~F}$ & 8 & Complex partial seizures (right or left temporal lobe origin) \\
\hline 6 & 29 & $\mathrm{~F}$ & 4 & Complex partial seizures (left temporal lobe origin) \\
\hline 7 & 45 & $\mathrm{~F}$ & 2 & Complex partial seizures (temporal lobe origin) \\
\hline 8 & 60 & M & 2 & Complex partial seizures (left temporal lobe origin) \\
\hline 9 & 39 & $\mathrm{~F}$ & 3 & Complex partial seizures (right hemisphere origin) \\
\hline 10 & 50 & $\mathrm{~F}$ & 3 & Complex partial seizures \\
\hline 11 & 60 & $\mathrm{~F}$ & 2 & Primary generalized epilepsy, non-convulsive status epilepticus \\
\hline 12 & 48 & M & 3 & Frontal lobe epilepsy \\
\hline 13 & 34 & $\mathrm{~F}$ & 9 & Lennox-Gastaut Syndrome (tonic seizures) \\
\hline 14 & 19 & M & 3 & $\begin{array}{l}\text { Primary generalized epilepsy or left frontal lobe seizures (tonic seizures with myoclonic jerks }+/ \text { - } \\
\text { secondary generalization) }\end{array}$ \\
\hline 15 & 32 & M & 12 & Complex partial seizures (right hemisphere origin) \\
\hline 16 & 35 & $\mathrm{~F}$ & 6 & Complex partial seizures (left hemisphere origin) \\
\hline 17 & 21 & $\mathrm{~F}$ & 5 & Lennox-Gastaut syndrome (tonic and atonic seizures) \\
\hline
\end{tabular}

Table 1: Participant demographics and characteristics of the epilepsy group.

\section{Heart rate and heart rate variability parameters}

The average heart rate and heart rate variability measures are summarized in Table 2 and Figure 1. Non-parametric related-samples testing demonstrated no significant changes in HR and HRV parameters shortly before epileptic seizures. During seizures, HR $(\mathrm{p}=0.003)$ and SDANN $(\mathrm{p}=0.005)$ significantly increased while VLF power significantly decreased $(\mathrm{p}=0.002)$. In the five-minute interval immediately following seizures, HR and SDANN were back to preseizure levels, while VLF power remained significantly decreased compared to the pre-seizure interval $(\mathrm{p}=0.009)$.
In the 5-minute interval preceding PNES, there was a significant increase in HR $(\mathrm{p}=0.037)$ and a significant decrease in SDANN $(\mathrm{p}=0.028)$ and VLF power $(\mathrm{p}=0.026)$. During PNES, there was a significant increase in HF power $(\mathrm{p}<0.001)$. In the interval following PNES, SDANN $(p=0.001)$ and VLF power $(p=0.001)$ were increased and $\operatorname{HR}(\mathrm{p}=0.037)$ was decreased, compared to the pre-seizure interval.

\begin{tabular}{|l|l|l|l|l|l|}
\hline & Interval -2 & Interval -1 & Interval i1 & Interval i2 & Interval +1 \\
\hline HR & & & & & \\
\hline Epilepsy & $75.0(12.1)$ & $75.1(12.1)$ & $79.8(14.6)$ & $79.2(11.0)$ & $78.3(9.9)$ \\
\hline PNES & $75.1(8.2)$ & $77.6(8.6)$ & $78.2(8.7)$ & $79.4(11.0)$ & $74.3(6.9)$ \\
\hline RMSSD & & & & & $0.033(0.02)^{* *}$ \\
\hline Epilepsy & $0.055(0.07)$ & $0.057(0.07)$ & $0.059(0.08)^{*}$ & $0.054(0.08)$ \\
\hline PNES & $0.074(0.05)$ & $0.066(0.05)$ & $0.081(0.05)$ & $0.085(0.06)$ & $0.068(0.06)$ \\
\hline
\end{tabular}


Citation: van der Kruijs SJM, Vonck KEJ, Feijs LMG, Bodde NMG, Lazeron RHC, et al. (2014) Autonomic Nervous System Functioning Associated with Epileptic Seizures: Analysis of Heart Rate Variability. J Neurol Neurophysiol 5: 215. doi:10.4172/2155-9562-5-1000215

Page 4 of 7

\begin{tabular}{|c|c|c|c|c|c|}
\hline \multicolumn{6}{|l|}{ SDANN } \\
\hline Epilepsy & $0.855(0.62)$ & $0.941(0.60)$ & $1.189(0.60)^{*}$ & $0.921(0.32)$ & $0.928(0.63)$ \\
\hline PNES & $0.817(0.09)$ & $0.787(0.08)$ & $0.797(0.09)$ & $0.789(0.11)$ & $0.835(0.11)$ \\
\hline \multicolumn{6}{|c|}{ VLF power } \\
\hline Epilepsy & $0.706(0.22)$ & $0.709(0.21)$ & $0.638(0.20)$ & $0.618(0.16)$ & $0.631(0.15)$ \\
\hline PNES & $0.685(0.15)$ & $0.634(0.12)$ & $0.655(0.16)$ & $0.649(0.17)$ & $0.722(0.20)$ \\
\hline \multicolumn{6}{|l|}{ LF power } \\
\hline Epilepsy & $0.0010(0.002)$ & $0.0011(0.001)$ & $0.0013(0.002)$ & $0.0006(0.000)$ & $0.0011(0.002)$ \\
\hline PNES & $0.0010(0.001)$ & $0.0015(0.002)$ & $0.0012(0.001)$ & $0.0013(0.001)^{\star}$ & $0.0011(0.001)$ \\
\hline \multicolumn{6}{|l|}{ HF power } \\
\hline Epilepsy & $0.0019(0.005)$ & $0.0023(0.005)$ & $0.0022(0.005)^{\star}$ & $0.001(0.001)^{\star \star}$ & $0.0019(0.005)$ \\
\hline PNES & $0.0026(0.004)$ & $0.0026(0.004)$ & $0.0032(0.003)$ & $0.003(0.004)$ & $0.0024(0.003)$ \\
\hline \multicolumn{6}{|c|}{ LF/HF ratio } \\
\hline Epilepsy & $1.820(1.12)$ & $1.927(1.42)$ & $1.894(1.13)$ & $2.408(2.24)^{*}$ & $2.176(1.34)$ \\
\hline PNES & $1.549(1.32)$ & $1.711(1.47)$ & $1.298(1.14)$ & $1.150(0.73)$ & $1.504(1.31)$ \\
\hline \multicolumn{6}{|c|}{$\begin{array}{ll}\begin{array}{l}\text { Poincaré } \\
\text { parameters }\end{array} & \text { plot }\end{array}$} \\
\hline \multicolumn{6}{|l|}{ SD1 } \\
\hline Epilepsy & $0.104(0.06)$ & $0.115(0.06)$ & $0.150(0.06)$ & $0.121(0.04)$ & $0.114(0.06)$ \\
\hline PNES & $0.120(0.06)$ & $0.132(0.07)$ & $0.163(0.05)$ & $0.152(0.06)$ & $0.127(0.06)$ \\
\hline \multicolumn{6}{|l|}{ SD2 } \\
\hline Epilepsy & $0.056(0.06)^{*}$ & $0.061(0.07)$ & $0.066(0.07)^{*}$ & $0.042(0.03)^{* *}$ & $0.058(0.07)$ \\
\hline PNES & $0.079(0.06)$ & $0.083(0.06)$ & $0.099(0.05)$ & $0.097(0.06)$ & $0.082(0.06)$ \\
\hline \multicolumn{6}{|c|}{$\mathrm{SD} 1 / \mathrm{SD} 2$ ratio } \\
\hline Epilepsy & $2.740(1.04)$ & $2.787(1.30)^{*}$ & $3.998(2.23)^{*}$ & $3.864(1.69)$ * & $3.219(1.57)$ \\
\hline PNES & $2.306(1.05)$ & $2.484(1.29)$ & $2.722(1.91)$ & $2.356(1.55)$ & $2.216(1.10)$ \\
\hline
\end{tabular}

Table 2: Descriptive statistics of heart rate and heart rate variability parameters. Results are reported as Mean (SD). Abbreviations: HR=heart rate, RMSSD=root mean square of successive differences, SDANN=standard deviation of average beat-to-beat intervals, VLF=very low frequency, $\mathrm{LF}=$ low frequency, $\mathrm{HF}=$ high frequency, $\mathrm{SD}=$ standard deviation. ${ }^{*}=$ Significant between-group differences for $\mathrm{p}<0.05 .{ }^{* *}=\mathrm{Significant}$ between-group differences for $\mathrm{p}<0.01$. Within-group differences are described in the text. 
Citation: van der Kruijs SJM, Vonck KEJ, Feijs LMG, Bodde NMG, Lazeron RHC, et al. (2014) Autonomic Nervous System Functioning Associated with Epileptic Seizures: Analysis of Heart Rate Variability. J Neurol Neurophysiol 5: 215. doi:10.4172/2155-9562-5-1000215

Page 5 of 7

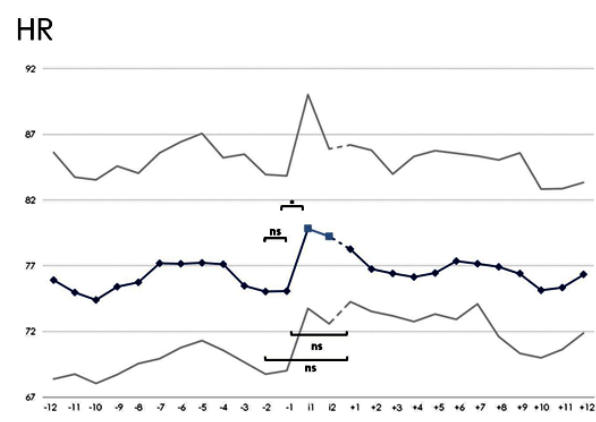

\section{SDANN}

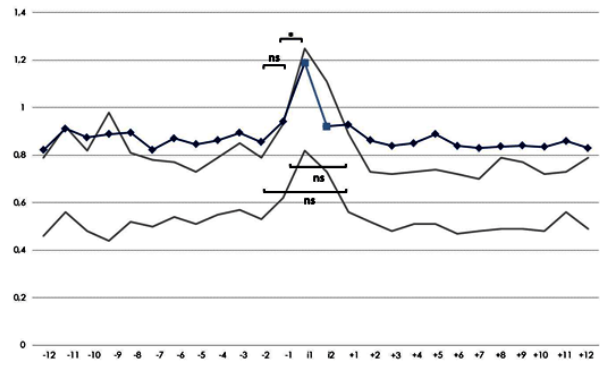

VLF power

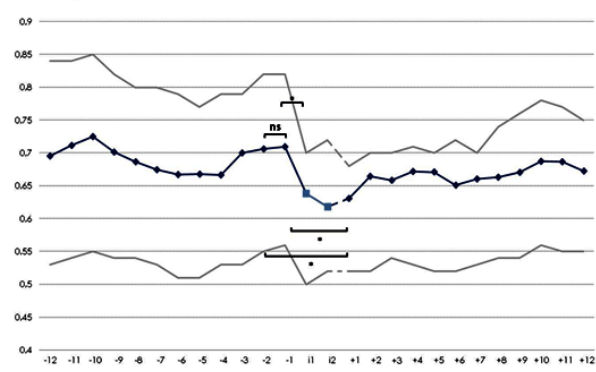

Figure 1: Average pattern of heart rate and linear heart rate variability indices which demonstrated significant peri-ictal changes in the epilepsy group. The blue line represents the average HR/HRV characteristic, with $95 \%$ confidence intervals in grey. Each interval lasted approximately 5 minutes: Intervals -12 to -1 comprise the hour preceding the seizure, the intervals i1 and i2 cover the seizure, and the hour following the seizure consists of the intervals +1 to +12 . The line between interval i 2 and +1 is dashed because not all patients had episodes consisting of exactly 2 intervals. * $=\mathrm{p}<0.05, \mathrm{~ns}=\mathrm{Not}$ significant. $\mathrm{HR}=$ heart rate, SDANN=standard deviation of average $\mathrm{NN}$ intervals, $\mathrm{VLF}=$ very low frequency.

\section{Poincaré plot parameters}

Non-parametric related-samples tests indicated no significant changes in Poincaré plot parameters shortly before epileptic seizures. During seizures, SD1 $(\mathrm{p}=0.005)$ and $\mathrm{SD} 1 / \mathrm{SD} 2$ ratio $(\mathrm{p}=0.01)$ significantly increased. In the five-minute interval immediately following seizures, SD1 decreased to pre-seizure levels, while SD1/SD2 ratio remained significantly increased compared to the pre-seizure interval $(\mathrm{p}=0.019)$.

In the first interval of PNES episodes, significant increases in SD1 $(p=0.010)$ and SD2 $(p=0.019)$ were observed. In the interval following PNES, the SD1/SD2 ratio significantly decreased when compared to the pre-seizure interval $(\mathrm{p}=0.044)$.

\section{Comparison between epileptic seizures and PNES episodes}

Non-parametric independent-samples tests demonstrated significantly higher pre-ictal SD1/SD2 ratios in patients with epilepsy compared to patients with PNES. Ictal RMSSD, HF power, LF power and SD2 were significantly higher in patients with PNES compared to patients with epilepsy. SDANN, LF/HF ratio and SD1/SD2 ratio were significantly higher in patients with epilepsy. No significant betweengroup differences were identified for the post-ictal HRV parameters.

\section{Discussion}

The current study examined differences in cardiac autonomic functioning associated with the occurrence of epileptic seizures. Cardiac measures were recorded during video-EEG monitoring. Significant ictal changes in HRV measures were identified: an average increase in heart rate, SDANN, SD1 and SD1/SD2 ratio and a decrease in VLF power were observed. In the five minutes directly after the seizure, heart rate, SDANN and SD1 returned to pre-seizure levels, while VLF power remained significantly decreased and SD1/SD2 ratio remained significantly increased compared to the interval preceding the seizure. No significant changes were identified in the pre-ictal interval or in intervals distant from the seizure.

Comparison with previously identified peri-ictal HRV patterns of patients with PNES resulted in significant between-group differences in pre-ictal SD2 and SD1/SD2 ratio and ictal RMSSD, SDANN, LF power, HF power, $\mathrm{LF} / \mathrm{HF}$ ratio, SD2 and SD1/SD2 ratio. No significant differences were identified in post-ictal HRV parameters.

\section{Clinical interpretation}

The ictal increase in heart rate suggests an increase in sympathetic functioning and reduced vagal tone during epileptic seizures, which is consistent with previous findings reported in literature [2,6]. In contrast, the Poincaré plot parameter SD1 increased, which is assumed to reflect elevated vagal tone. The increase in SDANN and decrease in VLF power are more difficult to interpret, since the exact physiological correlates are still unknown. However, it has been shown that SDANN is heavily influenced by physical activity [29-31], which may explain the ictal changes.

It has been suggested that the ictal reduction of the vagal tone may be one of the reasons for sudden unexpected death in epilepsy (SUDEP), although very few SUDEP cases have been reported during video-EEG (and ECG) monitoring and the exact mechanism leading to the seizure-related cardiac arrest remains unclear [8]. Specific factors may predispose patients with epilepsy to parasympathetic dysfunction and form possible reasons for extra supervision, such as use of carbamazepine [32].

\section{Implications for seizure prediction}

We did not identify substantial pre-ictal changes in heart rate and HRV parameters. This may be due to the fact that we examined 
averages over 5-minute intervals; we did not assess the dynamics of the change from the pre-ictal to the ictal HRV profile on ms-sec level. Our study can therefore not be compared with the findings of Jeppesen et al. [4], who identified specifically high HF power 10 seconds preceding seizure onset in three patients with temporal lobe epilepsy. Possibly, pre-ictal changes in HRV parameters only emerge seconds before the seizure and are therefore missed by our research design.

In contrast, findings of Behbahani et al. [7], do suggest that the 5minute interval preceding a seizure can be considered as prediction time for designing an algorithm of early detection of seizure onset based on HRV. They observed increased heart rate, LF/HF ratio and $\mathrm{SD} 1 / \mathrm{SD} 2$ ratio 30 minutes before the seizure, and an even stronger increase 5 minutes before the seizure, when compared to 240 minutes before the seizure. As a consequence, we must not exclude the potential of HRV parameters as measured on a 5-minute time-scale as seizure predictors; possibly other factors such as small $\mathrm{n}$ or heterogeneity of the study population have negatively influenced our ability to identify significant pre-ictal HRV changes. Future investigation of HRV changes measured in seconds or milliseconds and in larger patient populations would be particularly interesting for seizure detection uses.

\section{Implications for differential diagnosis}

Differential diagnosis between epilepsy and psychogenic nonepileptic seizures has been proven to be complicated; the period between the onset of PNES and the eventual diagnosis is often more than 6 years [33]. The initial misdiagnosis as epilepsy has serious consequences for patients with PNES, such as exposure to unnecessary antiepileptic medication, and also has a substantial societal burden, as it causes the patients to consume a disproportionate amount of scarce medical resources [34] and erroneous treatments for intractable epilepsy are expensive [35].

For these reasons, it is important to identify factors that can accurately differentiate between PNES and epilepsy. Ponnusamy et al. [6] were the first to compare the autonomic nervous system functioning of patients with epilepsy and patients with PNES. They identified significantly lower heart rate and higher RMSSD, LF power and HF power during PNES episodes compared with epileptic seizures, and demonstrated that the altered ictal HRV parameters could be used to differentiate epileptic seizures from PNES. Their findings are similar to the between-group differences that were identified in the current study, although their study design was not suited to draw conclusions about peri-ictal changes in HRV parameters. The results of the current study suggest that, in addition to ictal between-group differences, pre-ictal differences in HRV parameters may also be possible factors to distinguish epileptic seizures from PNES.

\section{Limitations}

We have carried out a relatively high number of statistical comparisons on a relatively modest dataset. The small study population may have led to type I errors (false negatives) due to lack of power, while the relatively large amount of comparisons may have resulted in type II errors (false positives). Also, the small $\mathrm{n}$ may have led several of the HRV averages to fall outside the $95 \%$ confidence interval, although the confidence intervals show the same trends as shown by the averages. Furthermore, the study population of patients with epilepsy was very heterogeneous, since multiple seizure types were included, each of which may have a distinctive influence on heart rate and heart rate variability. However, in view of the exploratory nature of this first study of the peri-ictal pattern of HRV parameters we thought it was appropriate to report our findings in this form.

\section{Conclusion}

Accepting the limitations, our data show that epileptic seizures are associated with increased heart rate, indicating an increased sympathetic tone and reduced vagal tone. HRV parameter changes are more difficult to interpret and need further investigation. The between-group differences that were identified between epileptic seizures and PNES even suggest that, after replication of our findings by future research, it may even be possible to identify peri-ictal HR(V) signatures with sufficient specificity to aid in the differential diagnosis between epilepsy and PNES.

\section{References}

1. Delamont RS, Julu PO, Jamal GA (1999) Changes in a measure of cardiac vagal activity before and after epileptic seizures. Epilepsy Res 35: 87-94.

2. Leutmezer F, Schernthaner C, Lurger S, Pötzelberger K, Baumgartner C (2003) Electrocardiographic changes at the onset of epileptic seizures. Epilepsia 44: 348-354.

3. Zaatreh MM, Quint SR, Tennison MB, D’Cruz O, Vaughn BB. (2003) Heart rate variability during interictal epileptiform discharges. Epilepsy Res 54: 85-90.

4. Jeppesen J, Beniczky S, Fuglsang-Frederiksen A, Sidenius P, Jasemian Y (2010) Detection of epileptic-seizures by means of power spectrum analysis of heart rate variability: a pilot study. Technol Health Care 18: 417-426.

5. Pradhan C, Sinha S, Thennarasu K, Jagadisha T (2011) Quantitative analysis of heart rate variability in patients with absence epilepsy. Neurol India 59: 25-29.

6. Ponnusamy A, Marques JL, Reuber M (2012) Comparison of heart rate variability parameters during complex partial seizures and psychogenic nonepileptic seizures. Epilepsia 53: 1314-1321.

7. Behbahani S, Dabanloo NJ, Nasrabadi AM, Teizeira CA, Dourado A (2013) Pre-ictal heart rate variability assessment of epileptic seizures by means of linear and non-linear analyses. Anadolu Kardiyol Derg 13: 797-803.

8. Tomson T, Nashef L, Ryvlin P (2008) Sudden unexpected death in epilepsy: current knowledge and future directions. Lancet Neurol 7: 1021-1031.

9. Surges R, Thijs RD, Tan HL, Sander JW (2009) Sudden unexpected death in epilepsy: risk factors and potential pathomechanisms. Nat Rev Neurol 5: 492-504.

10. Bodde NM, Brooks JL, Baker GA, Boon PA, Hendriksen JG, et al. (2009) Psychogenic non-epileptic seizures--Diagnostic issues: A critical review. Clin Neurol Neurosurg 111: 1-9.

11. Reuber M (2009) The etiology of psychogenic non-epileptic seizures: towards a biopsychosocial model. Neurol Clin 27: 909-924.

12. Malik M, Camm AJ, Kleiger RE (1996) Heart rate variability. Standards of measurement, physiological interpretation, and clinical use. Task Force of the European Society of Cardiology and the North American Society of Pacing and Electrophysiology. Eur Heart J 17: 354-381.

13. Kemp B, Olivan J. (2003) European data format 'plus'(EDF+), and EDF alike standard format for the exchange of physiological data. Clin Neurophysiol 114:1755-1761.

14. Akselrod S, Gordon D, Ubel FA, Shannon DC, Berger AC, et al. (1981) Power spectrum analysis of heart rate fluctuation: a quantitative probe of beat to beat cardiovascular control. Science 213: 220-222. 
Citation: van der Kruijs SJM, Vonck KEJ, Feijs LMG, Bodde NMG, Lazeron RHC, et al. (2014) Autonomic Nervous System Functioning Associated with Epileptic Seizures: Analysis of Heart Rate Variability. J Neurol Neurophysiol 5: 215. doi:10.4172/2155-9562-5-1000215

Page 7 of 7

15. Pomeranz M, Macaulay RJB, Caudill MA, Kutz I, Adam D et al. (1985) Assessment of autonomic function in humans by heart rate spectral analysis. Am J Physiol 248 :H151-153.

16. Malliani A, Pagani M, Lombardi F, Cerutti S (1991) Cardiovascular neural regulation explored in the frequency domain. Circulation 84 : 1482-1492.

17. Kamath MV, Fallen EL (1993) Power spectral analysis of heart rate variability: a noninvasive signature of cardiac autonomic function. Crit Rev Biomed Eng 21: 245-311.

18. Rimoldi O, Pierini S, Ferrari A, Cerutti S, Pagani M, et al. (1990) Analysis of short-term oscillations of R-R and arterial pressure in conscious dogs. Am J Physiol 258:H967-976.

19. Montano N, Ruscone TG, Porta A, Lombardi F, Pagani M, et al. (1994) Power spectrum analysis of heart rate variability to assess the changes in sympathovagal balance during graded orthostatic tilt. Circulation 90: 1826-1831.

20. Appel ML, Berger RD, Saul JP, Smith JM, Cohen RJ (1989) Beat to beat variability in cardiovascular variables: noise or music? J Am Coll Cardiol 14: 1139-1148.

21. Marciano F, Migaux ML, Acanfora D, Furgi C, Rengo F (1994) Quantification of Poincare maps for the evaluation of heart rate variability. Comp cardiol 557-580.

22. D'Addio G, Acanfora D, Pinna G, Maestri R, Furgi C, et al. (1998) Reproducibility of short- and long-term poincare plot parameters compared with frequency-domain HRV indexes in congestive heart failure. Comp cardiol 381-384.

23. Huikuri HV, Seppanen T, Koistinen M, Airaksinen J, Ikäheimo MJ, et al (1996) Abnormalities in beat-to-beat dynamics of heart rate before spontaneous onset of life-threatening ventricular tachyarrhythmias in patients with prior myocardial infarction. Circulation 93: 1836-1844.

24. Tulppo MP, Makikallio TH, Takala TE, Seppanen T, Huikuri HV (1996) Quantitative beat-to-beat analysis of heart rate dynamics during exercise. Am J Physiol 271: H244-252.
25. Kamen PW, Tonkin AM (1995) Application of the Poincaré plot to heart rate variability: a new measure of functional status in heart failure. Aust NZ J Med 25: 18-26.

26. Kamen PW, Krum H, Tonkin AM (1996) Poincaré plot of heart rate variability allows quantitative display of parasympathetic nervous activity. Clin Sci 91: 201-208.

27. Kamen PW (1996) Heart rate variability. Aus Fam Phys 25: 1087-1094.

28. Brunetto AF, Silva BM, Roseguini BT, Hirai DM, Guedes DP (2005) Limiar ventilatório e variabilidade da frequência cardíaca em adolescentes. Rev Bras Med Esporte 11: 22-27.

29. Roach D, Sheldon A, Wilson W, Sheldon R (1998) Temporally localized contributions to measures of large-scale heart rate variability. Am J Physiol 274: H1465-1471.

30. Roach D, Wilson W, Ritchie D, Sheldon R (2004) Dissection of longrange heart rate variability: controlled induction of prognostic measures by activity in the laboratory. J Am Coll Cardiol 43: 2271-2277.

31. Raj SR, Roach DE, Koshman ML, Sheldon RS (2004) Activity-responsive pacing produces long-term heart rate variability. J Cardiovasc Electrophysiol 15: 179-183.

32. Persson H, Ericson M, Tomson T (2003) Carbamazepine affects autonomic cardiac control in patients with newly diagnosed epilepsy. Epilepsy Res 57: 69-75.

33. Alsaadi TM, Marquez AV (2005) Psychogenic nonepileptic seizures. Am Fam Physician 2: 849-856.

34. Kirmayer L, Robbins J (1991) Three forms of somatization in primary care: prevalence, co-occurrence, and sociodemographic characteristics. J Nerv Ment Dis 179: 647-655.

35. Martin RC, Gilliam FG, Kilgore M, Faught E, Kuzniecky R (1998) Improved health care resource utilization following video-EEGconfirmed diagnosis of nonepileptic psychogenic seizures. Seizure 7: 385-390. 